\title{
Politique
}

Politique

\section{Dominique Boivin, Le lobbying ou le pouvoir des groupes de pressions, Montréal, Éditions du Méridien, 1984, 241 p.}

\section{Jean Dion}

Numéro 7, hiver 1985

Projection internationale du Québec

URI : https://id.erudit.org/iderudit/040485ar

DOI : https://doi.org/10.7202/040485ar

Aller au sommaire du numéro

Éditeur(s)

Société québécoise de science politique

ISSN

0711-608X (imprimé)

1918-6584 (numérique)

Découvrir la revue

Citer ce compte rendu

Dion, J. (1985). Compte rendu de [Dominique Boivin, Le lobbying ou le pouvoir des groupes de pressions, Montréal, Éditions du Méridien, 1984, 241 p.]

Politique, (7), 123-127. https://doi.org/10.7202/040485ar d'utilisation que vous pouvez consulter en ligne.

https://apropos.erudit.org/fr/usagers/politique-dutilisation/ 
Dominique Boivin, Le lobbying ou le pouvoir des groupes de pressions, Montréal, Éditions du Méridien, 1984, 241 p.

«Cet ouvrage n'est qu'un essai», souligne Dominique Boivin, après avoir précisé d'entrée de jeu que «tout essai vise la réussite». Et la réussite, dans la vie professionnelle d'un citoyen qui s'affiche ouvertement comme «conseiller en affaires gouvernementales» ou "démarcheur politique et parlementaire», réside essentiellement dans son pouvoir de conviction. Le lobbying s'annonce donc comme une vaillante tentative de démystification du phénomène-titre et un plaidoyer soutenu en faveur d'une reconnaissance virtuelle par les autorités étatiques de ceux qui exercent cette activité, encore un peu floue parce que mal structurée dans sa définition. Il ne s'agit point d'un panorama exhaustif de l'exercice du lobbying à travers le monde, mais davantage d'un essai à saveur polémique, comme l'est d'ailleurs intensément l'activité elle-même.

Dominique Boivin a étudié la science politique et, depuis une dizaine d'années, exerce à Québec, auprès de l'Assemblée nationale notamment, le bizarre métier de lobbyiste. Il est d'ailleurs l'un de ces très rares "professionnels de l'influence» à s'afficher ouvertement comme lobbyiste au Québec (et même au Canada) et à avoir pignon sur rue en cette qualité. Situation guère facile, on l'admettra aisément, que celle du lobbyiste qui, nos mœurs politiques étant ce qu'ils sont, voit son travail associé aux notions les plus sombres de la culture politique contemporaine: trafic d'influence, soudoiement, corruption, pouvoirs occultes, financement illicite, et quoi encore. C'est, par conséquent, dans une optique de correction et de rééquilibrage d'une pareille situation que se situe Le lobbying: réhabiliter et refaire une vertu à cet exercice qui va aux sources mêmes de la démocratie de représentation. Et l'apprivoisement de ce dernier-né des institutions politiques modernes passe par une nécessaire compréhension du phénomène, ce à quoi, en clair, s'attarde Boivin. 
Notre auteur semble accorder, dès les premières pages de sa dissertation, une mission particulière au lobbying et à sa reconnaissance de facto. Tout en s'efforçant de redonner aux grands malades du parlementarisme que sont le lobbying et ses praticiens l'image la plus juste possible, il constate également les sentiments «d'impuissance, de démobilisation et de désintéressement» caractéristiques de notre société à l'égard d'un pouvoir lointain et d'une fonction publique pléthorique, les pires dangers pouvant guetter un régime démocratique. À ce niveau, suggère-t-on, pourquoi ne pas considérer le lobbying, voix des groupes d'intérêt et pierre d'assise du fondamental droit de pétition enchâssé dans le First Amendment de la Constitution des États-Unis (où l'activité compte sur une meilleure réputation), comme le remède susceptible de rescaper une démocratie malade du gigantisme bureaucratique?

La première partie de l'ouvrage de Boivin est consacrée à la mise en forme d'une perspective générale quant au lobbying tel qu'il est pratiqué principalement au Québec, et à un survol de la façon dont il s'articule avec l'activité des groupes de pression. Le postulat d'attaque est celui qui veut que, d'une part, le lobbyiste ne s'impose pas uniquement en tant que défenseur des intérêts particuliers, mais qu'il peut fort bien exercer certaines représentations au nom de l'intérêt public (pour peu que cette expression puisse recouvrir une réalité précise) et que, contre toute attente, intérêts particuliers et bien général, d'autre part, ne sont pas nécessairement propositions antithétiques. Cette dernière idée va à l'encontre d'une certaine culture politique québécoise et canadienne, de forte inspiration anglo-saxonne, qui a contribué à entacher d'immoralité la poursuite d'activités de démarchage, au nom du caractère antidémocratique de politiques préférentielles initiées via certains contacts "privilégiés».

Boivin s'efforce d'abord de circonscrire une définition satisfaisante du concept de lobbying: il y constate le faible consensus 
quant à la portée exacte du terme, tout en faisant ressortir que, paradoxalement, l'on s'entend fort bien pour lui accoler une étiquette péjorative. Le terme "lobby» se voit fréquemment associé au qualificatif "puissant» lorsqu'un groupe particulièrement bien organisé fait montre de représentations soutenues auprès de l'appareil d'État; à l'inverse, on parlera de «justes revendications» lorsqu'il sera question, par exemple, de pressions de groupes de consommateurs. L'on constate l'ambivalence des mots, ainsi que la difficulté de faire la part des choses dans l'observation et l'analyse de «transactions verbales» aussi délicates.

Un second chapitre est consacré à une mise en scène destinée à illustrer dans sa quotidienneté le phénomène de la pression sociale et politique; il y est souligné que les groupes sont susceptibles, souvent, de naître spontanément et de se dissoudre lorsqu'un objectif ponctuel a été atteint. Boivin tente surtout de faire valoir que le lobbying, ou pression sur diverses instances décisionnelles, demeure potentiellement l'apanage de tout groupe qui, tôt ou tard, aura à y recourir s'il tient à être efficace dans son action. Le problème crée le groupe, et les bonnes démarches créent le groupe qui a du succès.

Les chapitres suivants s'attardent à démontrer combien, comme l'avait laissé entendre le titre, les notions de groupe de pression et de lobbying sont interdépendantes en système démocratique. L'auteur se livre alors à une analyse du phénomène sous tous ses aspects: les groupes de pression et leur constitution, leurs rapports avec une opinion publique changeante, leur relation et la concordance/discordance de leur action en regard de celle des partis d'opposition. De nombreuses statistiques concernant la surveillance de l'activité gouvernementale par les formations oppositionnistes à l'Assemblée nationale sour fournies (nombre d'heures consacrées à l'étude des crédits budgétaires, de séances consacrées à l'étude des dépenses), mais leur réelle utilité dans le cadre précis d'une 
discussion nettement sociologique reste à démontrer. L'on discerne toutefois, à travers ces tableaux, que Boivin désire souligner que les partis d'opposition ne font guère preuve d'efficacité dans l'accomplissement de leur tâche et, par là, faire la preuve que des groupes de pression au lobbying vigilant s'avèrent essentiels au maintien de la démocratie en marche.

Le chapitre 5, qui comprend aussi une sorte de "petit guide du parfait lobbyiste», et la conclusion sont les parties qui, du point de vue analytique, comportent les observations les plus intéressantes. Boivin y constate que les règles du jeu en matière de lobbying, ne serait-ce que par la volatilité des opinions, s'avèrent difficiles à suivre; l'argent prend une réputation de passe-partout par excellence, la corruption devient aux yeux des non-initiés de l'extérieur le mot d'ordre pour caractériser cette activité. Mais autant ces notions font-elles la vie dure au lobbyiste de bon aloi, autant notre auteur s'applique-t-il à détruire ces mythes et à en faire longuement ressortir le caractère exceptionnel.

Dominique Boivin conclut cette discussion principale en invoquant le sens de la démocratie pour officialiser le lobbying et ceux qui l'exercent; s'il importe d'offrir un contrepoids à un exécutif toujours plus puissant et à une bureaucratie toujours plus lourde et envahissante et devant lesquels le citoyen se sent constamment plus démuni, il importera aussi d'offrir aux groupes de meilleurs recours et des possibilités accrues. Dans cette optique, le lobbyiste reconnu, ayant pignon sur rue et identifié comme tel, ne pourra qu'être facteur de transparence dans les activités de démarchage des groupes, en plus d'être un agent d'information et un communicateur de premier plan susceptible de rapprocher les gouvernants de leurs commettants.

Un appendice (qui représente plus du tiers du volume) est d'ailleurs consacré à la reproduction d'un mémoire présenté à l'Assemblée nationale en octobre 1982 par Boivin lui-même, 
portant sur la reconnaissance d'un statut pour les lobbyistes. Le lobbying parlementaire y est présenté comme une nécessité et une solution, source de réelle démocratisation de nos institutions par l'accord d'une voix au chapitre pour les groupes d'intérêt, qui sont principe de représentativité, de légitimité et d'accès et de diffusion de l'information. Il ne pourra en résulter, conclut-on, qu'un accroissement du rôle de forum d'opinions qui doit être celui des institutions parlementaires. L'on peut cependant noter au passage que, en novembre dernier, l'Assemblée nationale, par la voix de son président Richard Guay, a décidé de surseoir indéfiniment à toute velléité de réglementation du lobbying, prétextant que rien pour l'instant ne justifie l'établissement d'une telle reconnaissance. Mauvais score pour un lobbyiste, qui ne parvient pas à réussir le sien propre...

Quoi qu'il en soit, cet ouvrage constitue un effort louable de démystification d'une activité déconsidérée parce que, à notre avis, méconnue. Il faut savoir gré à Dominique Boivin de s'efforcer d'apporter une plus grande transparence à la profession, bien qu'il importe de conserver à l'esprit que la démocratie derrière des portes closes (ce qu'est le lobbying) ne remportera jamais la faveur enthousiaste d'un public déjà suffisamment éloigné du pouvoir. Si le lobbying se conjugue avec le verbe pouvoir et lui est, en somme, parallèle, force nous est de faire une ultime constatation: les amitiés politiques, les bonnes fréquentations et les principes de réciprocité et de connivence ont de tout temps fait partie et seront toujours partie intégrante du jeu politique. À tout prendre, il est dans ces conditions préférable de savoir qui joue, avec quelles pièces, et d'avoir la possibilité de s'adjoindre un allié de premier calibre. 